\title{
On some shift invariant integral operators, univariate case
}

\author{
by George A. Anastassiou (Memphis, Tenn.) and \\ Heinz H. Gonska (Duisburg)
}

\begin{abstract}
In recent papers the authors studied global smoothness preservation by certain univariate and multivariate linear operators over compact domains. Here the domain is $\mathbb{R}$. A very general positive linear integral type operator is introduced through a convolution-like iteration of another general positive linear operator with a scaling type function. For it sufficient conditions are given for shift invariance, preservation of global smoothness, convergence to the unit with rates, shape preserving and preservation of continuous probabilistic functions. Finally, four examples of very general specialized operators are presented fulfilling all the above properties; in particular, the inequalities for global smoothness preservation are proven to be sharp.
\end{abstract}

1. Introduction. In approximating a function $f \in C(\mathbb{R})$ by means of approximation operators $\mathcal{L}_{k}$, it is interesting to examine which properties of $f$ are preserved by the approximants $\mathcal{L}_{k} f$. For example some of these could be: preservation of global smoothness given by the modulus of continuity, shape preservation, and preservation of properties of a probabilistic distribution function. Another important property of the operators $\mathcal{L}_{k}$ is the one of shift invariance. Also of interest is their convergence to the unit operator with rates.

In this article we introduce a very general family of operators $\mathcal{L}_{k}$ (see $\S 2)$ and we study the above characteristics by giving sufficient conditions so they hold, plus we give several examples of such operators. Our research has been mainly motivated by the works of Anastassiou, Cottin and Gonska

1991 Mathematics Subject Classification: 26A15, 26A18, 41A17, 41A25, 41A35, 41A36, 41A55, 41A99, 60E05.

Key words and phrases: global smoothness preservation, convergence to the unit with rates, Jackson type inequalities, sharp inequalities, modulus of continuity, integral operators, shift invariant operators, convolution type operators, shape preserving operators, probabilistic distribution function.

Research of both authors supported in part by NATO Grant CRG 891013. 
[1-2] and Anastassiou and $\mathrm{Yu}[3-4]$. We would like to mention some results from there.

Theorem A ([1]). Let $(X, d)$ be a compact metric space, and $L: C(X) \rightarrow$ $C(X), L \neq 0$, be a bounded linear operator mapping $\operatorname{Lip}(X)$ to $\operatorname{Lip}(X):=$ $\bigcup_{M>0} \operatorname{Lip}_{M}(1 ; X)$ such that for all $g \in \operatorname{Lip}(X)$,

$$
|L g|_{\text {Lip }} \leq c|g|_{\text {Lip }} \quad\left(|g|_{\text {Lip }}:=\sup _{d(x, y)>0} \frac{|g(x)-g(y)|}{d(x, y)}\right)
$$

with constant $c$ possibly depending on $L$, but independent of $g$. Then for all $F \in C(X)$ and $t \geq 0$,

$$
\omega_{1}(L f ; t) \leq\|L\| \widetilde{\omega}_{1}(f ; c t /\|L\|),
$$

where $\widetilde{\omega}_{1}$ is the least concave majorant of $\omega_{1}$.

In the univariate case we get

Theorem B ([1]). Let I be a compact interval, and $L: C(I) \rightarrow C(I)$, $L \neq 0$, be a bounded linear operator mapping $C^{1}(I)$ to $C^{1}(I)$. Then the estimate $(*)$ of Theorem $\mathrm{A}$ is true for all $f \in C(I)$ if the condition

$$
\left\|(L g)^{\prime}\right\|_{I} \leq c\left\|g^{\prime}\right\|_{I}
$$

is satisfied for all $g \in C^{1}(I)$.

In the multivariate case we obtain

Theorem C ([2]). Let $X$ be a compact convex subset of $\mathbb{R}^{k}$ equipped with the metric $d_{p}, 1 \leq p \leq \infty$, and let $L: C(X) \rightarrow C(X)$ be an operator satisfying the assumptions of Theorem $\mathrm{A}$. Then for all $f \in C(X)$ and $t \geq 0$ we have

$$
\omega_{1, d_{p}}(L f ; t) \leq(\|L\|+c) \omega_{1, d_{p}}(f ; t),
$$

where $\omega_{1, d_{p}}$ is the modulus of continuity with respect to $d_{p}$ and

$$
\begin{gathered}
d_{p}\left(\left(x_{1}, \ldots, x_{k}\right),\left(y_{1}, \ldots, y_{k}\right)\right)=\left(\sum_{i=1}^{k}\left|x_{i}-y_{i}\right|^{p}\right)^{1 / p}, \quad 1 \leq p<\infty \\
d_{\infty}\left(\left(x_{1}, \ldots, x_{k}\right),\left(y_{1}, \ldots, y_{k}\right)\right)=\max _{1 \leq i \leq k}\left|x_{i}-y_{i}\right| .
\end{gathered}
$$

Definition A. Let $\varphi$ be a bounded right-continuous function on $\mathbb{R}$ of compact support $\subseteq[-a, a], a>0$. Define

$$
\varphi_{k j}(x):=2^{k / 2} \varphi\left(2^{k} x-j\right) \quad \text { for } k, j \in \mathbb{Z} .
$$

For $f \in C(\mathbb{R})$, we define

$$
\left(\widetilde{A}_{k} f\right)(x):=\sum_{j=-\infty}^{\infty}\left\langle f, \varphi_{k j}\right\rangle \varphi_{k j}(x) \quad \text { for } k \in \mathbb{Z},
$$


where

$$
\left\langle f, \varphi_{k j}\right\rangle:=\int_{-\infty}^{\infty} f(t) \varphi_{k j}(t) d t .
$$

Note that $\widetilde{A}_{k}(f)(x)=\widetilde{A}_{0}\left(f\left(2^{-k} \cdot\right)\right)\left(2^{k} x\right)$.

TheOREM D ([3]). Assume

(i) $\sum_{j=-\infty}^{\infty} \varphi(x-j) \equiv 1, \forall x \in \mathbb{R}$;

(ii) there is a number $b \in \mathbb{R}$ such that $\varphi(x)$ is non-decreasing if $x \leq b$ and is non-increasing if $x \geq b$.

Let $F(x)$ be a continuous probabilistic distribution function on $\mathbb{R}$. Then $\widetilde{A}_{k}(F)$ is a probabilistic distribution function and satisfies

$$
\left|\widetilde{A}_{k}(F)(x)-F(x)\right| \leq \omega_{1}\left(F ; a / 2^{k-1}\right), \quad x \in \mathbb{R}, k \in \mathbb{Z} .
$$

Moreover, the above inequalities are sharp in the sense that the constant 1 in front of $\omega_{1}$ is the best possible.

Next, if $\varphi$ is continuous and as in Definition A, we have

TheOrem E ([4]). Assume

(i) $\sum_{j=-\infty}^{\infty} \varphi(x-j) \equiv 1$ on $\mathbb{R}$;

(ii) $\sum_{j=-\infty}^{\infty} j \varphi(x-j)$ is a linear function on $\mathbb{R}$;

(iii) there exist real numbers $b_{1}$ and $b_{2}, b_{1} \leq b_{2}$, such that $\varphi(x)$ is convex on $\left(-\infty, b_{1}\right]$ and $\left[b_{2},+\infty\right)$ respectively, and $\varphi(x)$ is concave on $\left[b_{1}, b_{2}\right]$.

Then, for $f \in C(\mathbb{R})$, if $f$ is a convex function on $\mathbb{R}, \widetilde{A}_{k}(f)$ is also convex on $\mathbb{R}$ and satisfy

$$
\left|\widetilde{A}_{k}(f)(x)-f(x)\right| \leq \omega_{1}\left(f ; a / 2^{k-1}\right), \quad x \in \mathbb{R}, k \in \mathbb{Z} .
$$

2. Main results. Let $X:=C_{\mathrm{U}}(\mathbb{R})$ be the space of uniformly continuous real valued functions on $\mathbb{R}$ and $C(\mathbb{R})$ the space of continuous functions from $\mathbb{R}$ into itself. For any $f \in X$ we have $\omega_{1}(f ; \delta)<+\infty, \delta>0$, where $\omega_{1}$ is the first modulus of continuity with respect to the supremum norm.

Let $\left\{l_{k}\right\}_{k \in \mathbb{Z}}$ be a sequence of positive linear operators that map $X$ into $C(\mathbb{R})$ with the property:

$$
l_{k}(f ; x)=l_{0}\left(f\left(2^{-k} \cdot\right) ; x\right), \quad x \in \mathbb{R}, f \in X .
$$

For fixed $a>0$ we assume that

$$
\sup _{\substack{u ; y \in \mathbb{R} \\|u-y| \leq a}}\left|l_{0}(f ; u)-f(y)\right| \leq \omega_{1}\left(f ; \frac{m a+n}{2^{r}}\right)
$$

for any $f \in X$, where $m \in \mathbb{N}, n \in \mathbb{Z}_{+}, r \in \mathbb{Z}$. 
Let $\varphi$ be a real valued function of compact support $\subseteq[-a, a], \varphi \geq 0, \varphi$ is Lebesgue measurable and such that

$$
\int_{-\infty}^{\infty} \varphi(x-u) d u=1, \quad \forall x \in \mathbb{R} .
$$

One can easily find that

$$
\int_{-\infty}^{\infty} \varphi(u) d u=1
$$

\section{EXAMPLES.}

$$
\varphi(x):=\chi_{[-1 / 2,1 / 2)}(x)= \begin{cases}1, & x \in[-1 / 2,1 / 2), \\ 0, & \text { elsewhere }\end{cases}
$$

the characteristic function;

$$
\varphi(x):= \begin{cases}1-x, & 0 \leq x \leq 1 \\ 1+x, & -1 \leq x \leq 0 \\ 0, & \text { elsewhere }\end{cases}
$$

the hat function.

Let $\left\{\mathcal{L}_{k}\right\}_{k \in \mathbb{Z}}$ be the sequence of positive linear operators acting on $X$ and defined by

$$
\mathcal{L}_{k}(f ; x):=\int_{-\infty}^{\infty} l_{k}(f ; u) \varphi\left(2^{k} x-u\right) d u
$$

In particular,

$$
\mathcal{L}_{0}(f ; x)=\int_{-\infty}^{\infty} l_{0}(f ; u) \varphi(x-u) d u .
$$

By (1) we observe that

$$
\mathcal{L}_{k}(f ; x)=\mathcal{L}_{0}\left(f\left(2^{-k} \cdot\right) ; 2^{k} x\right), \quad x \in \mathbb{R} .
$$

If the definition of $l_{k}$ is extended to $C(\mathbb{R})$ and $l_{k}$ maps $\pi_{n}$ into itself, then $\mathcal{L}_{k}$ does the same; here $\pi_{n}$ denotes the space of polynomials of degree at most $n \in \mathbb{Z}_{+}$. For the sake of convenience we make the following informal definition.

Definition 1. Let $f_{\alpha}(\cdot):=f(\cdot+\alpha), \alpha \in \mathbb{R}$, and $\phi$ be an operator. If $\phi\left(f_{\alpha}\right)=(\phi f)_{\alpha}$, then $\phi$ is called a shift invariant operator.

Proposition 1. Assume that

$$
l_{0}\left(f\left(2^{-k} \cdot+\alpha\right) ; 2^{k} u\right)=l_{0}\left(f\left(2^{-k} \cdot\right) ; 2^{k}(u+\alpha)\right),
$$

for all $k \in \mathbb{Z}, \alpha \in \mathbb{R}$ fixed, all $u \in \mathbb{R}$ and any $f \in X$. Then $\mathcal{L}_{k}$ is a shift invariant operator for all $k \in \mathbb{Z}$. 
Proof. Note that

$$
\left(\mathcal{L}_{0} f\right)(x)=\int_{-\infty}^{\infty}\left(l_{0} f\right)(u) \varphi(x-u) d u=\int_{-\infty}^{\infty}\left(l_{0} f\right)(x-u) \varphi(u) d u
$$

From (7) we have

$$
\begin{aligned}
\mathcal{L}_{k}(f(\cdot+\alpha) ; x) & =\mathcal{L}_{k}\left(f_{\alpha} ; x\right)=\mathcal{L}_{0}\left(f_{\alpha}\left(2^{-k} \cdot\right) ; 2^{k} x\right) \\
& =\int_{-\infty}^{\infty}\left(l_{0} f\left(2^{-k} \cdot+\alpha\right)\right)\left(2^{k} x-u\right) \varphi(u) d u \\
& =\int_{-\infty}^{\infty}\left(l_{0} f\left(2^{-k} \cdot+\alpha\right)\right)\left(2^{k}\left(x-2^{-k} u\right)\right) \varphi(u) d u \\
& =\int_{-\infty}^{\infty}\left(l_{0}\left(f\left(2^{-k} \cdot\right)\right)\right)\left(2^{k}\left(x-2^{-k} u+\alpha\right)\right) \varphi(u) d u \\
& =\mathcal{L}_{0}\left(f\left(2^{-k} \cdot\right) ; 2^{k}(x+\alpha)\right)=\mathcal{L}_{k}(f ; x+\alpha),
\end{aligned}
$$

i.e., $\mathcal{L}_{k}\left(f_{\alpha}\right)=\left(\mathcal{L}_{k}(f)\right)_{\alpha}$.

Next we study the property of global smoothness preservation of the operators $\mathcal{L}_{k}$.

TheOREM 1. For any $f \in X$ assume that, for all $u \in \mathbb{R}$,

$$
\left|l_{0}(f ; x-u)-l_{0}(f ; y-u)\right| \leq \omega_{1}(f ;|x-y|),
$$

for any $x, y \in \mathbb{R}$. Then

$$
\omega_{1}\left(\mathcal{L}_{k} f ; \delta\right) \leq \omega_{1}(f ; \delta)
$$

for any $\delta>0$.

Proof. Notice that

$$
\begin{aligned}
\left|\mathcal{L}_{0}(f ; x)-\mathcal{L}_{0}(f ; y)\right| & =\left|\int_{-\infty}^{\infty} l_{0}(f ; u) \varphi(x-u) d u-\int_{-\infty}^{\infty} l_{0}(f ; u) \varphi(y-u) d u\right| \\
& =\left|\int_{-\infty}^{\infty} l_{0}(f ; x-u) \varphi(u) d u-\int_{-\infty}^{\infty} l_{0}(f ; y-u) \varphi(u) d u\right| \\
& \leq \int_{-\infty}^{\infty} \varphi(u)\left|l_{0}(f ; x-u)-l_{0}(f ; y-u)\right| d u \\
& \leq\left(\int_{-\infty}^{\infty} \varphi(u) d u\right) \sup _{u \in \mathbb{R}}\left|l_{0}(f ; x-u)-l_{0}(f ; y-u)\right| \\
& \leq \omega_{1}(f ;|x-y|),
\end{aligned}
$$

by (4) and (9). 
From (7) we now have

$$
\begin{aligned}
\left|\mathcal{L}_{k}(f ; x)-\mathcal{L}_{k}(f ; y)\right| & =\left|\mathcal{L}_{0}\left(f\left(2^{-k} \cdot\right) ; 2^{k} x\right)-\mathcal{L}_{0}\left(f\left(2^{-k} \cdot\right) ; 2^{k} y\right)\right| \\
& \leq \omega_{1}\left(f\left(2^{-k} \cdot\right) ; 2^{k}|x-y|\right)=\omega_{1}(f ;|x-y|),
\end{aligned}
$$

i.e., global smoothness of $\mathcal{L}_{k}$ has been established.

The convergence of $\mathcal{L}_{k}$ to $I$ as $k \rightarrow+\infty$ ( $I$ is the unit operator) with rates is studied in the following:

TheOREM 2. For $f \in X$, under the assumption (2), we have

$$
\left|\mathcal{L}_{k}(f ; x)-f(x)\right| \leq \omega_{1}\left(f ; \frac{m a+n}{2^{k+r}}\right),
$$

where $m \in \mathbb{N}, n \in \mathbb{Z}_{+}, k, r \in \mathbb{Z}$.

Proof. From $(7),(3)$ and $\operatorname{supp} \varphi \subseteq[-a, a]$, we observe that

$$
\begin{aligned}
\left|\mathcal{L}_{k}(f ; x)-f(x)\right|= & \left|\mathcal{L}_{0}\left(f\left(2^{-k} \cdot\right) ; 2^{k} x\right)-f\left(2^{-k}\left(2^{k} x\right)\right)\right| \\
= & \left|\int_{-\infty}^{\infty}\left[l_{0}\left(f\left(2^{-k} \cdot\right) ; u\right)-f\left(2^{-k}\left(2^{k} x\right)\right)\right] \varphi\left(2^{k} x-u\right) d u\right| \\
= & \left|\int_{2^{k} x-a}^{2^{k} x+a}\left[l_{0}\left(f\left(2^{-k} \cdot\right) ; u\right)-f\left(2^{-k}\left(2^{k} x\right)\right)\right] \varphi\left(2^{k} x-u\right) d u\right| \\
\leq & 2_{2^{k} x-a \leq u \leq 2^{k} x+a}^{\sup _{0}}\left|l_{0}\left(f\left(2^{-k} \cdot\right) ; u\right)-f\left(2^{-k}\left(2^{k} x\right)\right)\right| \\
& \times\left(\int_{2^{k} x-a}^{2^{k} x+a} \varphi\left(2^{k} x-u\right) d u\right),
\end{aligned}
$$

i.e.,

(12) $\left|\mathcal{L}_{k}(f ; x)-f(x)\right| \leq \sup _{2^{k} x-a \leq u \leq 2^{k} x+a}\left|l_{0}\left(f\left(2^{-k} \cdot\right) ; u\right)-f\left(2^{-k}\left(2^{k} x\right)\right)\right|$.

Consider $g:=f\left(2^{-k} \cdot\right) \in X$. Hence the right-hand side of (12) equals

$$
\sup _{\left|u-2^{k} x\right| \leq a}\left|l_{0}(g ; u)-g\left(2^{k} x\right)\right| \leq \omega_{1}\left(g ; \frac{m a+n}{2^{r}}\right)=\omega_{1}\left(f ; \frac{m a+n}{2^{k+r}}\right),
$$

the last inequality being valid by (2). Thus we have established (11).

Remark 1. (i) Assume that $\partial\left(l_{0} f\right)(x-u) / \partial x$ exists and is continuous in $x, u$ and $\varphi(u)$ is continuous in $u$, for $x \in \mathbb{R}, u \in[-a, a]$. Then, from (6) by the Leibniz rule, we get

$$
\frac{d\left(\mathcal{L}_{0} f\right)}{d x}(x)=\int_{-a}^{a}\left(l_{0} f\right)_{x}(x-u) \varphi(u) d u .
$$


Thus $\left(\mathcal{L}_{k} f\right)(x)=\left(\mathcal{L}_{0} f\left(2^{-k} \cdot\right)\right)\left(2^{k} x\right), k \in \mathbb{Z}$, is also differentiable.

(ii) Let $i \geq 1$ be an integer, assume that $\partial^{i-1}\left(l_{0} f\right)(x-u) / \partial x^{i-1}$ and $\partial^{i}\left(l_{0} f\right)(x-u) / \partial x^{i}$ exist and are continuous in $x, u$ and $\varphi(u)$ is continuous in $u$, for $x \in \mathbb{R}, u \in[-a, a]$. Then

$$
\frac{d^{i}\left(\mathcal{L}_{0} f\right)}{d x^{i}}(x)=\int_{-a}^{a} \frac{\partial^{i}\left(l_{0} f\right)}{\partial x^{i}}(x-u) \varphi(u) d u,
$$

i.e., $\mathcal{L}_{k}$ is $i$ times differentiable.

(iii) Assuming that $l_{0}$ maps $X^{(i)}$ (the space of $i$ times continuously differentiable functions from $X$ ) into $C^{(i)}(\mathbb{R})$, the space of $i$ times continuously differentiable functions from $\mathbb{R}$ into itself, we see that if $f \in X^{(i)}$ then $\partial^{i-1}\left(l_{0} f\right)(x-u) / \partial x^{i-1}$ and $\partial^{i}\left(l_{0} f\right)(x-u) / \partial x^{i}$ are continuous in $x$, $u$, where $i \geq 1$ is an integer.

We need the following

LEMMA 1. If $F(x, u)$ is continuous on $\mathbb{R} \times[-a, a]$, then $\int_{-a}^{a} F(x, u) d u$ is continuous in $x$.

\section{Pr o of. Trivial.}

Remark 2. (i) By Lemma 1, we see that if $l_{0}$ maps $X^{(i)}$ into $C^{(i)}(\mathbb{R})$, $\varphi$ being continuous on $[-a, a]$, then $\mathcal{L}_{k}$ maps also $X^{(i)}$ into $C^{(i)}(\mathbb{R})$, where $i \geq 0$ is an integer.

(ii) Since $\varphi \geq 0$ and $\varphi$ is continuous on $[-a, a]$ we have: if

$$
\frac{\partial^{i}\left(l_{0} f\right)}{\partial x^{i}}(x-u) \geq 0, \quad i \geq 1 \text { integer }
$$

then

$$
\frac{d^{i}\left(\mathcal{L}_{0} f\right)}{d x^{i}}(x) \geq 0
$$

(iii) Again assume that $\varphi$ is continuous on $[-a, a]$. One can easily prove that if $f^{(i)} \geq 0$ and $\frac{d^{i}}{d x^{i}} \mathcal{L}_{0}(f ; x) \geq 0$ for some $i \geq 1$, then

$$
\frac{d^{i}}{d x^{i}} \mathcal{L}_{k}(f ; x) \geq 0, \quad k \in \mathbb{Z}
$$

Note that if $l_{0} f$ is an increasing continuous function and $\varphi \geq 0$ is continuous on $[-a, a]$, one can easily see tha $\mathcal{L}_{0} f$ is increasing and continuous.

The above motivates the following

PROBLEM 1. If $f$ and $l_{0} f$ are continuous (probabilistic) distribution functions and $\varphi \geq 0$ is continuous on $[-a, a]$, can we conclude then that $\mathcal{L}_{0} f$ is a continuous distribution function?

Note that any continuous distribution function is in $X:=C_{\mathrm{U}}(\mathbb{R})$. The answer to this problem is in the affirmative, see Theorem 4 which follows. 
To prove Theorem 4 we need

Definition 2. Let $G, \psi$ be functions from $\mathbb{R}^{2}, \mathbb{R}$ (respectively) to $\mathbb{R}$. We say that

$$
\lim _{y \rightarrow y_{0}} G(x, y)=\psi(x)
$$

uniformly with respect to $x$ iff for every $\varepsilon>0$, there is $\delta>0$ such that for all $x$, if $\left|y-y_{0}\right|<\delta$ then $|G(x, y)-\psi(x)|<\varepsilon\left(|y|>\delta\right.$ if $\left.y_{0}= \pm \infty\right)$.

The next is a well-known basic result of Real Analysis.

Theorem 3. Assume that $\int_{\alpha}^{\beta} G(x, y) d x$ exists for all $y \in \mathbb{R}$. If $\lim _{y \rightarrow y_{0}} G(x, y)=\psi(x)\left(y_{0}\right.$ can be $\left.\pm \infty\right)$ uniformly with respect to $x$, then

$$
\lim _{y \rightarrow y_{0}} \int_{\alpha}^{\beta} G(x, y) d x=\int_{\alpha}^{\beta} \psi(x) d x=\int_{\alpha}^{\beta} \lim _{y \rightarrow y_{0}} G(x, y) d x .
$$

Again, here we assume that $\varphi \geq 0$ is continuous on $[-a, a], \operatorname{supp} \varphi \subseteq$ $[-a, a], \int_{-\infty}^{\infty} \varphi(x-u) d u=1$ for all $x \in \mathbb{R}$, and $f$ and $l_{0} f$ are distribution functions that are continuous from $\mathbb{R}$ into $\mathbb{R}$. Obviously by (1), $l_{k} f$ is a continuous distribution function too. We aim to prove that

$$
\left(\mathcal{L}_{k} f\right)(x)=\int_{-a}^{a} G(x, u) d u, \quad k \in \mathbb{Z}
$$

is a continuous distribution function, where

$$
G(x, u):=\left(l_{k} f\right)\left(2^{k} x-u\right) \varphi(u) .
$$

Note that $G$ is continuous in $(x, u) \in \mathbb{R} \times[-a, a]$, hence by Lemma $1, \mathcal{L}_{k} f$ is also continuous, and

$$
\lim _{x \rightarrow \pm \infty} G(x, u)=\varphi(u), 0 \quad \text { (respectively) } \forall u \in[-a, a] .
$$

Still we need to prove that

$$
\lim _{x \rightarrow \pm \infty} \int_{-a}^{a} G(x, u) d u=\int_{-a}^{a} \lim _{x \rightarrow \pm \infty} G(x, u) d u=1,0
$$

(respectively). The last is true from the next Lemmas 2 and 3, (4) and Theorem 3.

Lemma 2. $\lim _{x \rightarrow+\infty} G(x, u)=\varphi(u)$, uniformly with respect to $u$.

Proof. Let $\varepsilon>0$ be given, and observe that

$$
|G(x, u)-\varphi(u)|=|\varphi(u)| \cdot\left|\left(l_{k} f\right)\left(2^{k} x-u\right)-1\right| .
$$

Case (i): $|u|>a$. Then $|G(x, u)-\varphi(u)|=0$, by $\operatorname{supp} \varphi \subseteq[-a, a]$. 
Case (ii): $|u| \leq a$. Here $|\varphi(u)| \leq A$. Then

$$
\begin{aligned}
|G(x, u)-\varphi(u)| & =|\varphi(u)| \cdot\left|\left(l_{k} f\right)\left(2^{k} x-u\right)-1\right| \\
& \leq A\left|\left(l_{k} f\right)\left(2^{k} x-u\right)-1\right|=A\left(1-\left(l_{k} f\right)\left(2^{k} x-u\right)\right) \\
& \leq A\left(1-\left(l_{k} f\right)\left(2^{k} x-a\right)\right),
\end{aligned}
$$

by $l_{k} f$ being a distribution function.

Also

$$
\lim _{z \rightarrow+\infty}\left(l_{k} f\right)(z)=1,
$$

i.e., for $\varepsilon>0$ there is a $\bar{\delta}$ (real) such that $z>\bar{\delta}$ implies $1-\left(l_{k} f\right)(z)<\varepsilon$. Now choose $\delta$ (for the uniform convergence) as equal to $(\bar{\delta}+a) / 2^{k}$. Then for $x>\delta$ one has $2^{k} x-a>\bar{\delta}$. Hence

$$
1-\left(l_{k} f\right)\left(2^{k} x-a\right)<\varepsilon, \quad \forall x>\delta
$$

(for $x \rightarrow+\infty$ ). Now take $\varepsilon / A$ instead of $\varepsilon$, and do the same things again.

LEMMA $3 . \lim _{x \rightarrow-\infty} G(x, u)=0$, uniformly with respect to $u$.

$\operatorname{Pr}$ o of. Let $\varepsilon>0$ be given, and observe that

$$
|G(x, u)-0|=|\varphi(u)| \cdot\left|\left(l_{k} f\right)\left(2^{k} x-u\right)\right| .
$$

Case (i): $|u|>a$. Then $G(x, u)=0$.

Case (ii): $|u| \leq a$. Here $|\varphi(u)| \leq A$. Then

$$
\begin{aligned}
|G(x, u)| & =|\varphi(u)| \cdot\left|\left(l_{k} f\right)\left(2^{k} x-u\right)\right| \\
& \leq A\left|\left(l_{k} f\right)\left(2^{k} x-u\right)\right|=A\left(l_{k} f\right)\left(2^{k} x-u\right) \\
& \leq A\left(l_{k} f\right)\left(2^{k} x+a\right),
\end{aligned}
$$

by $l_{k} f$ being a distribution function.

We know that

$$
\lim _{z \rightarrow-\infty}\left(l_{k} f\right)(z)=0,
$$

i.e., for $\varepsilon>0$ there is a $\bar{\delta}>0$ such that $z<-\bar{\delta}$ implies $\left(l_{k} f\right)(z)<\varepsilon$. Now choose $\delta=(\bar{\delta}+a) / 2^{k}>0$. Then for $x<-\delta$ one has $2^{k} x+a<-\bar{\delta}$. Thus

$$
\left(l_{k} f\right)\left(2^{k} x+a\right)<\varepsilon, \quad \forall x<-\delta .
$$

Now take $\varepsilon / A$ instead of $\varepsilon$, and repeat the same things.

Now our final main result has been proven and is stated next.

THEOREM 4. Let $l_{k}$ be positive linear operators from $X$ into $C(\mathbb{R})$ as in (1) and $f$ be a (probabilistic) distribution function from $\mathbb{R}$ into itself that is continuous. Assume that $l_{k} f$ is also a continuous distribution function. Assume furthermore that $\varphi \geq 0$ is continuous on $[-a, a], a>0, \operatorname{supp}(\varphi) \subseteq$ 
$[-a, a]$, and $\int_{-\infty}^{\infty} \varphi(x-u) d u=1$ for all $x \in \mathbb{R}$. Then the operator

$$
\mathcal{L}_{k}(f ; x)=\int_{-a}^{a}\left(l_{k} f\right)\left(2^{k} x-u\right) \varphi(u) d u, \quad k \in \mathbb{Z},
$$

which is the same as in (5), when applied to $f$ as above produces a continuous (probabilistic) distribution function from $\mathbb{R}$ into itself.

3. Applications. Next we present four examples of shift invariant integral operators where $l_{k}$ is specified.

It will be shown that they satisfy exactly the theory presented in $\S 2$. The basic function $\varphi$ will always be as in $\S 2$. In particular, for the operators $\left(A_{k}\right)_{k \in \mathbb{Z}}$, to be defined next, $\varphi$ will be assumed additionally to be an even continuous function. The properties of our specific operators will be presented according to the order of the properties of the general operators $\mathcal{L}_{k}, k \in \mathbb{Z}$, in $\S 2$.

For each $k \in \mathbb{Z}$, we define

(i)

$$
\left(A_{k} f\right)(x):=\int_{-\infty}^{\infty} r_{k}^{f}(u) \varphi\left(2^{k} x-u\right) d u
$$

where

$$
r_{k}^{f}(u):=2^{k} \int_{-\infty}^{\infty} f(t) \varphi\left(2^{k} t-u\right) d t
$$

is continuous in $u$, i.e., here

$$
l_{k}(f ; u)=r_{k}^{f}(u), \quad u \in \mathbb{R} .
$$

(ii)

$$
\left(B_{k} f\right)(x):=\int_{-\infty}^{\infty} f\left(u / 2^{k}\right) \varphi\left(2^{k} x-u\right) d u
$$

i.e., here

$$
l_{k}(f ; u)=f\left(u / 2^{k}\right)
$$

is continuous in $u \in \mathbb{R}$.

(iii)

$$
\left(L_{k} f\right)(x):=\int_{-\infty}^{\infty} c_{k}^{f}(u) \varphi\left(2^{k} x-u\right) d u
$$


where

$$
c_{k}^{f}(u):=2^{k} \int_{2^{-k} u}^{2^{-k}(u+1)} f(t) d t
$$

is continuous in $u$, i.e., here

$$
l_{k}(f ; u)=c_{k}^{f}(u), \quad u \in \mathbb{R} .
$$

(iv)

$$
\left(\Gamma_{k} f\right)(x):=\int_{-\infty}^{\infty} \gamma_{k}^{f}(u) \varphi\left(2^{k} x-u\right) d u
$$

where

$$
\gamma_{k}^{f}(u):=\sum_{j=0}^{n} w_{j} f\left(\frac{u}{2^{k}}+\frac{j}{2^{k} n}\right), \quad n \in \mathbb{N}, w_{j} \geq 0, \sum_{j=0}^{n} w_{j}=1,
$$

i.e., here

$$
l_{k}(f ; u)=\gamma_{k}^{f}(u)
$$

is continuous in $u \in \mathbb{R}$.

First observe that (1) is satisfied by all $l_{k}$ (see (22), (24), (27), (30)) corresponding to the operators $A_{k}, B_{k}, L_{k}, \Gamma_{k}, k \in \mathbb{Z}$. Thus (7) is true for all $A_{k}, B_{k}, L_{k}, \Gamma_{k}$ :

$$
\begin{aligned}
& A_{k}(f ; x)=A_{0}\left(f\left(2^{-k} \cdot\right) ; 2^{k} x\right), \\
& B_{k}(f ; x)=B_{0}\left(f\left(2^{-k} \cdot\right) ; 2^{k} x\right), \\
& L_{k}(f ; x)=L_{0}\left(f\left(2^{-k} \cdot\right) ; 2^{k} x\right), \\
& \Gamma_{k}(f ; x)=\Gamma_{0}\left(f\left(2^{-k} \cdot\right) ; 2^{k} x\right), \quad \text { for all } k \in \mathbb{Z} .
\end{aligned}
$$

Note that

$$
A_{k}(1)=B_{k}(1)=L_{k}(1)=\Gamma_{k}(1)=1 .
$$

In case the above specific operators are defined on the whole $C(\mathbb{R})$ and if $\pi_{n}$ is the space of polynomials of degree $\leq n$, then

$$
A_{k}\left(\pi_{n}\right) \subseteq \pi_{n}, \quad B_{k}\left(\pi_{n}\right) \subseteq \pi_{n}, \quad L_{k}\left(\pi_{n}\right) \subseteq \pi_{n}, \quad \Gamma_{k}\left(\pi_{n}\right) \subseteq \pi_{n} .
$$

Proposition 2. $A_{k}, B_{k}, L_{k}, \Gamma_{k}$ are shift invariant operators.

Proof. Here we apply Proposition 1.

(i) For $A_{k}$ operators ( $\varphi$ even): Note that

$$
l_{0}(f ; x)=r_{0}^{f}(x)=\int_{-\infty}^{\infty} f(t) \varphi(t-x) d t
$$




$$
=\int_{-\infty}^{\infty} f(t) \varphi(x-t) d t=\int_{-\infty}^{\infty} f(x-t) \varphi(t) d t .
$$

Thus for $a \in \mathbb{R}$ we have

$$
\begin{aligned}
l_{0}\left(f\left(2^{-k} \cdot+a\right) ; 2^{k} u\right) & =\int_{-\infty}^{\infty} f\left(2^{-k}\left(2^{k} u-t\right)+a\right) \varphi(t) d t \\
& =\int_{-\infty}^{\infty} f\left(u+a-2^{-k} t\right) \varphi(t) d t \\
& =\int_{-\infty}^{\infty} f\left(2^{-k} 2^{k}(u+a)-2^{-k} t\right) \varphi(t) d t \\
& =\int_{-\infty}^{\infty} f\left(2^{-k}\left[2^{k}(u+a)-t\right]\right) \varphi(t) d t \\
& =l_{0}\left(f\left(2^{-k} \cdot\right) ; 2^{k}(u+a)\right) .
\end{aligned}
$$

Here $r_{0}^{f}$ satisfies (8). Therefore $A_{k}$ is a shift invariant operator.

(ii) For $B_{k}$ operators: Here $l_{0} f=f$. Thus

$$
\begin{aligned}
l_{0}\left(f\left(2^{-k} \cdot+a\right) ; 2^{k} u\right) & =f\left(2^{-k} 2^{k} u+a\right) \\
& =f(u+a)=l_{0}\left(f\left(2^{-k} \cdot\right) ; 2^{k}(u+a)\right) .
\end{aligned}
$$

(iii) For $L_{k}$ operators: Here

$$
l_{0}(f, x)=\int_{x}^{x+1} f(t) d t
$$

Then

$$
\begin{aligned}
l_{0}\left(f\left(2^{-k} \cdot+a\right) ; 2^{k} u\right) & =\int_{2^{k} u}^{2^{k} u+1} f\left(2^{-k} t+a\right) d t \\
& =\int_{2^{k}(u+a)}^{2^{k}(u+a)+1} f\left(2^{-k} t\right) d t=l_{0}\left(f\left(2^{-k} \cdot\right) ; 2^{k}(u+a)\right) .
\end{aligned}
$$

(iv) For $\Gamma_{k}$ operators:

$$
l_{0}(f ; u)=\sum_{j=0}^{n} w_{j} f\left(u+\frac{j}{n}\right) .
$$


Thus

$$
\begin{aligned}
l_{0}\left(f\left(2^{-k} \cdot+a\right) ; 2^{k} u\right) & =\sum_{j=0}^{n} w_{j} f\left(2^{-k}\left(2^{k} u+j / n\right)+a\right) \\
& =\sum_{j=0}^{n} w_{j} f\left(2^{-k}\left(2^{k}(u+a)+j / n\right)\right) \\
& =l_{0}\left(f\left(2^{-k} \cdot\right) ; 2^{k}(u+a)\right) .
\end{aligned}
$$

Next we show that the operators $A_{k}, B_{k}, L_{k}, \Gamma_{k}$ have the property of global smoothness preservation.

Theorem 5. For all $f \in C_{\mathrm{U}}(\mathbb{R})$ and all $\delta>0$ we have

$$
\begin{array}{ll}
\omega_{1}\left(A_{k} f ; \delta\right) \leq \omega_{1}(f ; \delta), & \omega_{1}\left(B_{k} f ; \delta\right) \leq \omega_{1}(f ; \delta), \\
\omega_{1}\left(L_{k} f ; \delta\right) \leq \omega_{1}(f ; \delta), & \omega_{1}\left(\Gamma_{k} f ; \delta\right) \leq \omega_{1}(f ; \delta) .
\end{array}
$$

Proof. Here we apply Theorem 1. It is enough to prove (9).

(i) For $A_{k}$ operators ( $\varphi$ even): From (34) we have

$$
\begin{aligned}
\left|l_{0}(f ; x-u)-l_{0}(f ; y-u)\right| & =\left|\int_{-\infty}^{\infty}(f(x-u-t)-f(y-u-t)) \varphi(t) d t\right| \\
& \leq \int_{-\infty}^{\infty}|f(x-u-t)-f(y-u-t)| \varphi(t) d t \\
& \leq \int_{-\infty}^{\infty} \omega_{1}(f ;|x-y|) \varphi(t) d t=\omega_{1}(f ;|x-y|),
\end{aligned}
$$

where the last equality holds by (4).

(ii) For $B_{k}$ operators: Here $l_{0} f=f$, therefore

$\left|l_{0}(f ; x-u)-l_{0}(f ; y-u)\right|=|f(x-u)-f(y-u)| \leq \omega_{1}(f ;|x-y|)$.

(iii) For $L_{k}$ operators: We have

$$
\begin{aligned}
\left|l_{0}(f ; x-u)-l_{0}(f ; y-u)\right| & =\left|c_{0}^{f}(x-u)-c_{0}^{f}(y-u)\right| \\
& =\left|\int_{x-u}^{x-u+1} f(t) d t-\int_{y-u}^{y-u+1} f(t) d t\right| \\
& =\left|\int_{0}^{1} f(w-u+x) d w-\int_{0}^{1} f(w-u+y) d w\right| \\
& \leq \int_{0}^{1}|f(w-u+x)-f(w-u+y)| d w \\
& \leq \omega_{1}(f ;|x-y|) .
\end{aligned}
$$


238

G. A. Anastassiou and H. H. Gonska

(iv) For $\Gamma_{k}$ operators: We have

$$
\begin{aligned}
\left|l_{0}(f ; x-u)-l_{0}(f ; y-u)\right| & =\left|\gamma_{0}^{f}(x-u)-\gamma_{0}^{f}(y-u)\right| \\
& =\left|\sum_{j=0}^{n} w_{j}(f(x-u+j / n)-f(y-u+j / n))\right| \\
& \leq \sum_{j=0}^{n} w_{j}|f(x-u+j / n)-f(y-u+j / n)| \\
& \leq \omega_{1}(f ;|x-y|) \sum_{j=0}^{n} w_{j}=\omega_{1}(f ;|x-y|) .
\end{aligned}
$$

THEOREM 6. Inequalities (35) are sharp, in the sense that they hold as equalities when $f(x)=x \in C_{\mathrm{U}}(\mathbb{R})$.

Proof. (i) For $A_{k}$ operators ( $\varphi$ even): Note that

$$
\begin{aligned}
\left(A_{k} f\right)(x) & =\int_{-\infty}^{\infty} r_{k}^{f}(u) \varphi\left(2^{k} x-u\right) d u \\
& =\int_{-\infty}^{\infty} r_{k}^{f}\left(2^{k} x-u\right) \varphi(u) d u=\int_{-a}^{a} r_{k}^{f}\left(2^{k} x-u\right) \varphi(u) d u .
\end{aligned}
$$

Here

$$
\begin{aligned}
r_{k}^{f}(u) & =2^{k} \int_{-\infty}^{\infty} f(t) \varphi\left(2^{k} t-u\right) d t=2^{k} \int_{-\infty}^{\infty} f(t) \varphi\left(u-2^{k} t\right) d t \\
& =\int_{-\infty}^{\infty} f\left(\frac{\tau}{2^{k}}\right) \varphi(u-\tau) d \tau=\int_{-\infty}^{\infty} f\left(\frac{u-\tau}{2^{k}}\right) \varphi(\tau) d \tau \\
& =\int_{-a}^{a} f\left(\frac{u-\tau}{2^{k}}\right) \varphi(\tau) d \tau .
\end{aligned}
$$

Therefore

$$
r_{k}^{f}\left(2^{k} x-u\right)=\int_{-a}^{a} f\left(x-\frac{u+\tau}{2^{k}}\right) \varphi(\tau) d \tau .
$$

If $f(x)=x$ then

$$
\begin{aligned}
r_{k}^{f}\left(2^{k} x-u\right)-r_{k}^{f}\left(2^{k} y\right. & -u) \\
& =\int_{-a}^{a}\left(f\left(x-\frac{u+\tau}{2^{k}}\right)-f\left(y-\frac{u+\tau}{2^{k}}\right)\right) \varphi(\tau) d \tau \\
& =\int_{-a}^{a}(x-y) \varphi(\tau) d \tau=x-y
\end{aligned}
$$


by (4). Thus

$$
\begin{aligned}
\left(A_{k} f\right)(x)-\left(A_{k} f\right)(y) & =\int_{-a}^{a}\left(r_{k}^{f}\left(2^{k} x-u\right)-r_{k}^{f}\left(2^{k} y-u\right)\right) \varphi(u) d u \\
& =\int_{-a}^{a}(x-y) \varphi(u) d u=x-y
\end{aligned}
$$

proving that $\omega_{1}\left(A_{k}(\mathrm{id}) ; \delta\right)=\omega_{1}(\mathrm{id} ; \delta), \delta>0$, where id stands for the identity map.

(ii) For $B_{k}$ operators: Note that

$$
\begin{aligned}
\left(B_{k} f\right)(x) & =\int_{-\infty}^{\infty} f\left(\frac{u}{2^{k}}\right) \varphi\left(2^{k} x-u\right) d u \\
& =\int_{-\infty}^{\infty} f\left(\frac{2^{k} x-u}{2^{k}}\right) \varphi(u) d u=\int_{-a}^{a} f\left(x-\frac{u}{2^{k}}\right) \varphi(u) d u
\end{aligned}
$$

When $f(x)=x$ we get

$$
\begin{aligned}
\left(B_{k} f\right)(x)-\left(B_{k} f\right)(y) & =\int_{-a}^{a}\left(f\left(x-\frac{u}{2^{k}}\right)-f\left(y-\frac{u}{2^{k}}\right)\right) \varphi(u) d u \\
& =\int_{-a}^{a}(x-y) \varphi(u) d u=x-y
\end{aligned}
$$

(iii) For $L_{k}$ operators: Note that

$$
\begin{aligned}
\left(L_{k} f\right)(x) & =\int_{-\infty}^{\infty} c_{k}^{f}(u) \varphi\left(2^{k} x-u\right) d u \\
& =\int_{-\infty}^{\infty} c_{k}^{f}\left(2^{k} x-u\right) \varphi(u) d u=\int_{-a}^{a} c_{k}^{f}\left(2^{k} x-u\right) \varphi(u) d u
\end{aligned}
$$

Here

$$
c_{k}^{f}(u)=2^{k} \int_{2^{-k} u}^{2^{-k}(u+1)} f(t) d t=2^{k} \int_{0}^{2^{-k}} f\left(t+\frac{u}{2^{k}}\right) d t
$$

that is,

$$
c_{k}^{f}\left(2^{k} x-u\right)=2^{k} \int_{0}^{2^{-k}} f\left(t+x-\frac{u}{2^{k}}\right) d t .
$$


If $f(x)=x$, then

$$
\begin{aligned}
c_{k}^{f}\left(2^{k} x-u\right)-c_{k}^{f}\left(2^{k} y-u\right) & =2^{k} \int_{0}^{2^{-k}}(x-y) d t \\
& =(x-y) 2^{k} \int_{0}^{2^{-k}} d t=x-y,
\end{aligned}
$$

and so again

$$
\left(L_{k} f\right)(x)-\left(L_{k} f\right)(y)=\int_{-a}^{a}(x-y) \varphi(u) d u=x-y .
$$

(iv) For $\Gamma_{k}$ operators: As before we obtain

$$
\Gamma_{k}(f ; x)=\sum_{j=0}^{n} w_{j} \int_{-\infty}^{\infty} f\left(x-\frac{u}{2^{k}}+\frac{j}{2^{k} n}\right) \varphi(u) d u .
$$

If $f(x)=x$, then

$$
\begin{aligned}
\Gamma_{k}(f ; x)-\Gamma_{k}(f ; y) & =\sum_{j=0}^{n} w_{j} \int_{-\infty}^{\infty}(x-y) \varphi(u) d u \\
& =(x-y)\left(\sum_{j=0}^{n} w_{j}\right) \int_{-\infty}^{\infty} \varphi(u) d u=x-y
\end{aligned}
$$

The operators $A_{k}, B_{k}, L_{k}, \Gamma_{k}, k \in \mathbb{Z}$, converge to the unit operator $I$ with rates as given below.

TheOREM 7. For $k \in \mathbb{Z}$,

$$
\begin{aligned}
& \left|A_{k}(f ; x)-f(x)\right| \leq \omega_{1}\left(f ; \frac{a}{2^{k-1}}\right), \\
& \left|B_{k}(f ; x)-f(x)\right| \leq \omega_{1}\left(f ; \frac{a}{2^{k}}\right), \\
& \left|L_{k}(f ; x)-f(x)\right| \leq \omega_{1}\left(f ; \frac{a+1}{2^{k}}\right),
\end{aligned}
$$

and

$$
\left|\Gamma_{k}(f ; x)-f(x)\right| \leq \omega_{1}\left(f ; \frac{a+1}{2^{k}}\right) .
$$

Proof. (i) For $A_{k}$ operators ( $\varphi$ even): By (34) and (3),

$$
\sup _{\substack{u, y \in \mathbb{R} \\|u-y| \leq a}}\left|l_{0}(f, u)-f(y)\right|
$$




$$
\begin{aligned}
& =\sup _{\substack{u, y \in \mathbb{R} \\
|u-y| \leq a}}\left|\int_{-\infty}^{\infty} f(t) \varphi(u-t) d t-\int_{-\infty}^{\infty} f(y) \varphi(u-t) d t\right| \\
& \leq \sup _{\substack{u, y \in \mathbb{R} \\
|u-y| \leq a}} \int_{-\infty}^{\infty}|f(t)-f(y)| \varphi(u-t) d t \\
& \leq \sup _{\substack{u, y \in \mathbb{R} \\
|u-y| \leq a}} \int_{-\infty}^{\infty} \omega_{1}(f ;|t-y|) \varphi(u-t) d t \\
& =\omega_{1}(f ; 2 a) \int_{-\infty}^{\infty} \varphi(u-t) d t=\omega_{1}(f ; 2 a) .
\end{aligned}
$$

proving (2). Hence by Theorem 2, we obtain (36).

(ii) For $B_{k}$ operators: Here $l_{0}(f, u)=f(u)$ and

$$
\sup _{\substack{u, y \in \mathbb{R} \\|u-y| \leq a}}\left|l_{0}(f ; u)-f(y)\right|=\sup _{\substack{u, y \in \mathbb{R} \\|u-y| \leq a}}|f(u)-f(y)|=\omega_{1}(f ; a),
$$

and we use Theorem 2.

(iii) For $L_{k}$ operators: Here

$$
\begin{aligned}
\sup _{\substack{u, y \in \mathbb{R} \\
|u-y| \leq a}}\left|l_{0}(f, u)-f(y)\right| & =\sup _{\substack{u, y \in \mathbb{R} \\
|u-y| \leq a}}\left|\int_{u}^{u+1} f(t) d t-f(y)\right| \\
& \leq \sup _{\substack{u, y \in \mathbb{R} \\
|u-y| \leq a}} \int_{u}^{u+1}|f(t)-f(y)| d t \\
& \leq \sup _{\substack{u, y \in \mathbb{R} \\
|u-y| \leq a}} \int_{u}^{u+1} \omega_{1}(f ;|t-y|) d t \\
& =\sup _{\substack{u, y \in \mathbb{R} \\
|u-y| \leq a}} \int_{0}^{1} \omega_{1}(f ;|t+u-y|) d t \\
& \leq \sup _{\substack{u, y \in \mathbb{R} \\
|u-y| \leq a}} \int_{0}^{1} \omega_{1}(f ; t+|u-y|) d t \\
& \leq \sup _{\substack{u, y \in \mathbb{R} \\
|u-y| \leq a}} \omega_{1}(f ; 1+|u-y|)=\omega_{1}(f ; 1+a),
\end{aligned}
$$

and we use Theorem 2 again. 
(iv) For $\Gamma_{k}$ operators: Here

$$
\begin{aligned}
\sup _{\substack{u, y \in \mathbb{R} \\
|u-y| \leq a}}\left|l_{0}(f, u)-f(y)\right| & =\sup _{\substack{u, y \in \mathbb{R} \\
|u-y| \leq a}}\left|\sum_{j=0}^{n} w_{j} f(u+j / n)-f(y)\right| \\
& \leq \sup _{\substack{u, y \in \mathbb{R} \\
|u-y| \leq a}} \sum_{j=0}^{n} w_{j}|f(u+j / n)-f(y)| \\
& \leq \sup _{\substack{u, y \in \mathbb{R} \\
|u-y| \leq a}} \sum_{j=0}^{n} w_{j} \omega_{1}(f ;|u+j / n-y|) \\
& \leq \sup _{\substack{u, y \in \mathbb{R} \\
|u-y| \leq a}} \sum_{j=0}^{n} w_{j} \omega_{1}(f ; j / n+|u-y|) \\
& \leq\left(\sum_{j=0}^{n} w_{j}\right) \omega_{1}(f ; 1+a)=\omega_{1}(f ; a+1) .
\end{aligned}
$$

Again, an application of Theorem 2 completes the proof.

Some final comments follow.

Remark 3. According to Remark 2 we have:

(i) Here we assume $\varphi \geq 0, \varphi$ continuous on $[-a, a], \operatorname{supp} \varphi \subseteq[-a, a]$. Let $f \in X^{(i)}, i \geq 0$ an integer. If $f^{(i)} \geq 0$, then

$$
\left(r_{0}^{f}\right)^{(i)} \geq 0, \quad\left(c_{0}^{f}\right)^{(i)} \geq 0, \quad\left(\gamma_{0}^{f}\right)^{(i)} \geq 0
$$

and so

$$
\left(A_{0} f\right)^{(i)} \geq 0, \quad\left(B_{0} f\right)^{(i)} \geq 0, \quad\left(L_{0} f\right)^{(i)} \geq 0, \quad\left(\Gamma_{0} f\right)^{(i)} \geq 0 .
$$

(ii) If $f^{(i)} \geq 0, i \geq 0$ an integer, $\varphi \geq 0, \varphi$ continuous on $[-a, a], \operatorname{supp} \varphi \subseteq$ $[-a, a]$, then

$\left(A_{k} f\right)^{(i)} \geq 0, \quad\left(B_{k} f\right)^{(i)} \geq 0, \quad\left(L_{k} f\right)^{(i)} \geq 0, \quad\left(\Gamma_{k} f\right)^{(i)} \geq 0, \quad$ for any $k \in \mathbb{Z}$.

An application of Theorem 4 comes next:

(iii) Let $\varphi \geq 0, \varphi$ continuous on $[-a, a], \operatorname{supp} \varphi \subseteq[-a, a]$,

$$
\int_{-\infty}^{\infty} \varphi(x-u) d u=1, \quad \forall x \in \mathbb{R} .
$$

Then the operators $A_{k}, B_{k}, L_{k}, \Gamma_{k}(k \in \mathbb{Z})$ map continuous probabilistic distribution functions into continuous probabilistic distribution functions.

Acknowledgements. The authors gratefully acknowledge the hospitality of the Mathematical Research Institute at Oberwolfach where this article was started and finished during two most pleasant visits. 


\section{References}

[1] G. Anastassiou, C. Cottin and H. Gonska, Global smoothness of approximating functions, Analysis 11 (1991), 43-57.

[2] - , - - , Global smoothness preservation by multivariate approximation operators, in: Israel Mathematical Conference Proc. 4, Weizmann Science Press, 1991, 31-44.

[3] G. Anastassiou and X. M. Yu, Monotone and probabilistic wavelet approximation, Stochastic Anal. Appl. 10 (1992), 251-264.

[4] -, -, Convex and coconvex-probabilistic wavelet approximation, ibid., 507-521.

DEPARTMENT OF MATHEMATICAL SCIENCES

THE UNIVERSITY OF MEMPHIS

MEMPHIS, TENNESSEE 38152, U.S.A.
DEPARTMENT OF MATHEMATICS UNIVERSITY OF DUISBURG D-47048 DUISBURG, GERMANY

Reçu par la Rédaction le 20.4.1993

Révisé le 15.10.1994 